DE DE GRUYTER

OPEN

G

BULGARIAN ACADEMY OF SCIENCES

CYBERNETICS AND INFORMATION TECHNOLOGIES • Volume 14, No 2

Sofia • 2014

Print ISSN: 1311-9702; Online ISSN: 1314-4081

DOI: $10.2478 /$ cait-2014-0021

\title{
Colour Restoration of Image Obtained From CCD Sensor Directly
}

\author{
Jun Luo*, Ying Chen** \\ *College of Science, Huazhong Agricultural University, 430070, Wuhan, Hubei Province, China \\ **Hubei Province Fiber Inspection Bureau, 430060, Wuhan, Hubei Province, China \\ Emails:1uojun81@163.com cy2047@163.com
}

Abstract: The original image data obtained from Charge-coupled Device (CCD) can be called original data, which is lack of color information. In order to restore the color of original image, firstly, we design a Bayer color filter array, and then we use bilinear interpolation algorithm and smooth hue transition interpolation algorithm to restore the color of original image. However, the hues of adjacent pixels change abruptly by the bilinear interpolation, therefore, we use smooth hue transition interpolation to enhance the edge of original image, and finally we identify the ultimate performance of these interpolation algorithms.

Keywords: Color restoration, CCD sensor, Bayer, Color filter array, Bilinear interpolation algorithm, Smooth hue transition interpolation algorithm, Edge enhancement, Objective quality measure.

\section{Introduction}

CCD chip does not record any real colour information, in order to restore the colour information of original image, usually, we apply three-CCD or single-CCD system to obtain colour image alternatively. In three-CCD system, we obtain red (R), green (G), blue (B) information by three CCD respectively, then we can restore a colourful image by RGB model. However, the price of this system is so expensive that we can not apply it in reality. Therefore, it is essential for us to use single-CCD 
camera, which apply interpolation algorithm to restore the colour information of original object [1-4]. In single-CCD system, we need to place a colour filter array (CFA) before the CCD chip. Each value of CFA stands for one pixel corresponding to the colour of R, G or B, so we need to restore the other two colour values of every pixel. There are many image interpolation algorithms to restore colour information, such as the nearest neighbour interpolation algorithm, bilinear interpolation algorithm [5-7].

In this paper, we will introduce the imaging model for the first, then give some introduction of interpolation algorithms, and finally identify the ultimate performance of these algorithms by several experiments.

\section{The model of single-CCD imaging}

Usually, every electronic signal unit of CCD is corresponding to the intensity of image pixel; if the values of pixels are all defined between 0 and 255, and then the intensity information of the pixels will stand for the real detail of original object.

We use a CFA in front of the CCD to rearrange the colour components for original image at first, then one pixel can just obtain one colour component, such as $\mathrm{R}, \mathrm{G}$, or B component, therefore, we need to compute the other two colour components by interpolation algorithms [8-10]. In accord with the model of singleCCD imaging, it is essential for us to pay attention to the CFA and interpolation algorithms.

There are several colour filter arrays for imaging application, such as Bayer CFA, Mosaic CFA and Strip CFA. As can be seen in Fig. 1(a), the starting pixels at odd rows of Bayer CFA are $\mathrm{R}$ and $\mathrm{G}$ components, and starting pixels at even rows are $G$ and $B$ components, besides, in Fig.1(b), the starting pixels at odd rows of Mosaic CFA are R, G and B components, and starting pixels at even rows are G, B and R components, in Fig.1(c), the starting pixels at first column of Strip CFA is R, and starting pixels at second column is $\mathrm{G}$, and at third column is B component [1].

\begin{tabular}{|c|c|c|c|c|c|}
\hline R & G & R & G & R & G \\
\hline G & B & G & B & G & B \\
\hline R & G & R & G & R & G \\
\hline G & B & G & B & G & B \\
\hline R & G & R & G & R & G \\
\hline G & B & G & B & G & B \\
\hline
\end{tabular}

(a)

\begin{tabular}{|c|c|c|c|c|c|}
\hline $\mathrm{R}$ & $\mathrm{G}$ & $\mathrm{B}$ & $\mathrm{G}$ & $\mathrm{R}$ & $\mathrm{B}$ \\
\hline $\mathrm{G}$ & $\mathrm{B}$ & $\mathrm{R}$ & $\mathrm{B}$ & $\mathrm{G}$ & $\mathrm{R}$ \\
\hline $\mathrm{R}$ & $\mathrm{G}$ & $\mathrm{B}$ & $\mathrm{G}$ & $\mathrm{R}$ & $\mathrm{B}$ \\
\hline $\mathrm{G}$ & $\mathrm{B}$ & $\mathrm{R}$ & $\mathrm{B}$ & $\mathrm{G}$ & $\mathrm{R}$ \\
\hline $\mathrm{R}$ & $\mathrm{G}$ & $\mathrm{B}$ & $\mathrm{G}$ & $\mathrm{R}$ & $\mathrm{B}$ \\
\hline $\mathrm{G}$ & $\mathrm{B}$ & $\mathrm{R}$ & $\mathrm{B}$ & $\mathrm{G}$ & $\mathrm{R}$ \\
\hline
\end{tabular}

(b)

\begin{tabular}{|c|c|c|c|c|c|}
\hline $\mathrm{R}$ & $\mathrm{G}$ & $\mathrm{B}$ & $\mathrm{R}$ & $\mathrm{G}$ & $\mathrm{B}$ \\
\hline $\mathrm{R}$ & $\mathrm{G}$ & $\mathrm{B}$ & $\mathrm{R}$ & $\mathrm{G}$ & $\mathrm{B}$ \\
\hline $\mathrm{R}$ & $\mathrm{G}$ & $\mathrm{B}$ & $\mathrm{R}$ & $\mathrm{G}$ & $\mathrm{B}$ \\
\hline $\mathrm{R}$ & $\mathrm{G}$ & $\mathrm{B}$ & $\mathrm{R}$ & $\mathrm{G}$ & $\mathrm{B}$ \\
\hline $\mathrm{R}$ & $\mathrm{G}$ & $\mathrm{B}$ & $\mathrm{R}$ & $\mathrm{G}$ & $\mathrm{B}$ \\
\hline $\mathrm{R}$ & $\mathrm{G}$ & $\mathrm{B}$ & $\mathrm{R}$ & $\mathrm{G}$ & $\mathrm{B}$ \\
\hline
\end{tabular}

(c)

Fig. 1. Bayer CFA (a); mosaic CFA (b); strip CFA (c)

Naturally, we would like to see green information because we can distinguish more details of original object at green component, as can be seen in Fig.1, the Bayer CFA supplies the $50 \%$ green component, and $25 \%$ red and blue components of whole CFA respectively, and the Mosaic CFA and Strip CFA supply 33.3\% red, blue and green component respectively, therefore, the Bayer CFA is better than 
Mosaic CFA and Strip CFA in accord with the human visual system, and we will analyse the CCD data corresponding to the Bayer CFA.

Usually, the data obtained directly from the CCD without any modification by the processing units of camera is called original data, which can reflect the light intensity of every pixel. However, the original data can not be displayed directly because they are not colour image in some degree. In this paper, we use Imagingsource camera DFK41BU02 made in Germany to obtain original image. As can be seen in Fig. 2(a), the original data of a key is stored by the camera, which is an 8-bit data file; each pixel of the original data represents the greyscale of $R, G$, or $B$ value between 0 and 255. In Fig. 2(b), we convert the Fig. 2(a) to a 24-bit image in accord with the Bayer CFA, and we can see the enlargement image of the red box area of Fig. 2(b) in Fig. 2(c). In order to identify the performance of our algorithms, we obtain another original data of little metal bear by the same camera, which can be seen in Fig. 3(a), (b) and (c).

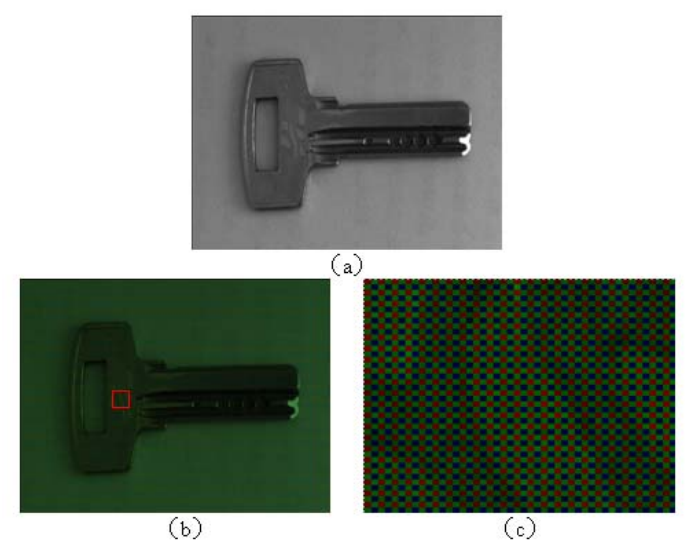

Fig. 2. The original data of a key (a); convert the Fig. 2(a) to a 24-bit image in accord with Bayer CFA (b); the enlargement image of the red box area in Fig. 2(b) (c)

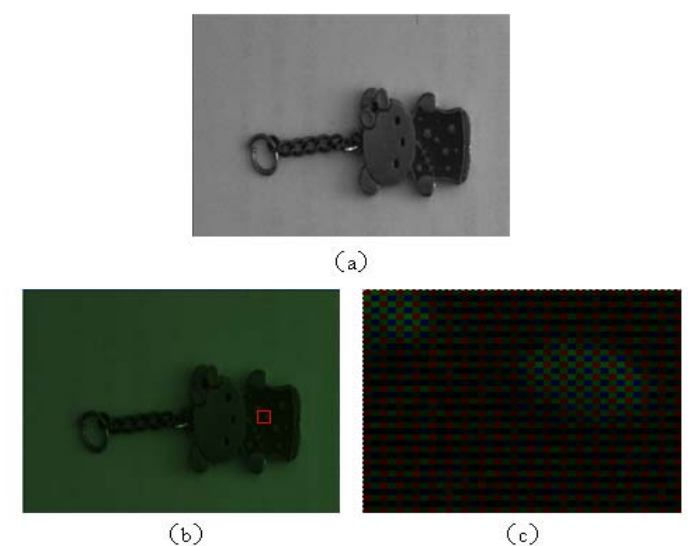

Fig. 3. The original data of a little metal bear (a); convert the Fig. 3(a) to a 24-bit image in accord with Bayer CFA (b); the enlargement image of the red box area in Fig. 3(b) (c) 
In order to restore the colour information of Fig. 2(a) and Fig. 3(a), we need to calculate the other two colour components of every pixel. In accord with the Bayer imaging model, we can restore the colour information by two steps. For the first, we will design a Bayer CFA starting with two particular pixels for original data, and then we apply a suitable colour interpolation algorithm corresponding to the Bayer CFA to restore the other two colour components of original data.

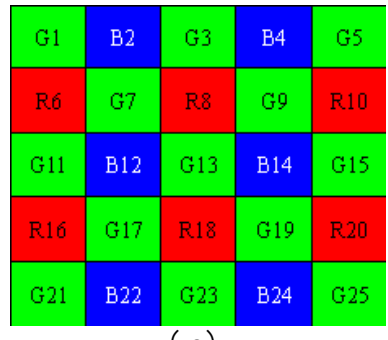

( a)

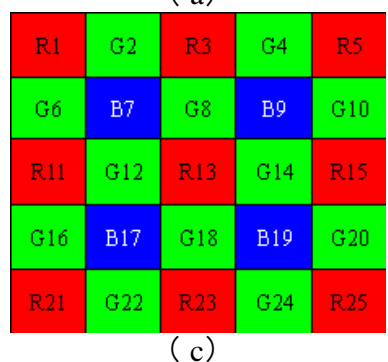

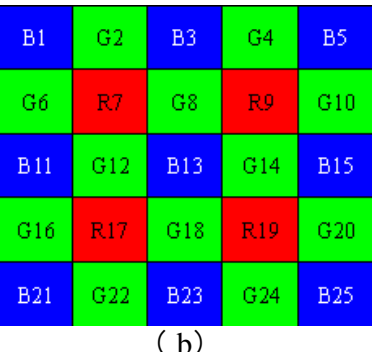

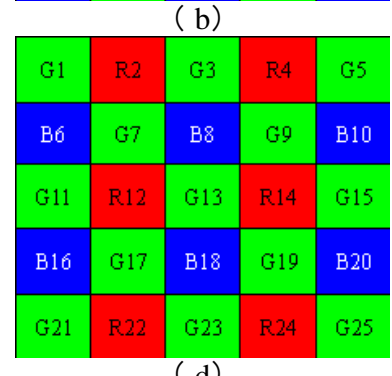

(d)

Fig. 4. The Bayer colour filter array models starting with: GB pixels (a); BG pixels (b); RG pixels (c); and GR pixels (d)

We can see four Bayer CFAs starting with GB, BG, RG and GR pixels in Fig. 4, the pixel named G1, B2 and R6 represents the green, blue and red value of the first, second and sixth pixel respectively. The different start pixels CFA needs different interpolation algorithms, but only one CFA is correct in accord with the specific camera.

\section{The principle of interpolation algorithms}

\subsection{Bilinear interpolation algorithm for original data}

According to the Bayer CFA depicted in Fig. 4, we use bilinear interpolation algorithm to restore the colour information of original data by following three methods.

\section{- Interpolation of green pixels}

The average of the upper, lower, left and right pixel values is assigned as the $G$ value of the interpolated pixel. For example, in Fig. 4(a), we can obtain the values of G8 at R8, and G12 at B12 in (1):

$$
\left\{\begin{aligned}
\mathrm{G} 8 & =(\mathrm{G} 3+\mathrm{G} 7+\mathrm{G} 9+\mathrm{G} 13) / 4 \\
\mathrm{G} 12 & =(\mathrm{G} 7+\mathrm{G} 11+\mathrm{G} 13+\mathrm{G} 17) / 4
\end{aligned}\right.
$$




\section{- Interpolation of red/blue pixels at a green position}

The average of two adjacent pixel values in corresponding colour is assigned to the interpolated pixel. For example, in Fig. 4(a), we can obtain the values of B7 and R7 at G7 in (2), and B13 and R13 at G13 in (3):

$$
\left\{\begin{array}{l}
\mathrm{B} 7=(\mathrm{B} 2+\mathrm{B} 12) / 2, \\
\mathrm{R} 7=(\mathrm{R} 6+\mathrm{R} 8) / 2 .
\end{array}\right.
$$

At G13, it corresponds to the B13 and R13 values for

$$
\left\{\begin{array}{c}
\mathrm{B} 13=(\mathrm{B} 12+\mathrm{B} 14) / 2, \\
\mathrm{R} 13=(\mathrm{R} 8+\mathrm{R} 18) / 2 .
\end{array}\right.
$$

- Interpolation of a red/blue pixel at a blue/red position

The average of four adjacent diagonal pixel values is assigned to the interpolated pixel. For example, in Fig. 4(a), we can obtain the values of B8 at R8, and R12 at B12 in (4):

$$
\left\{\begin{array}{c}
\mathrm{B} 8=(\mathrm{B} 2+\mathrm{B} 4+\mathrm{B} 12+\mathrm{B} 14) / 4, \\
\mathrm{R} 12=(\mathrm{R} 6+\mathrm{R} 8+\mathrm{R} 16+\mathrm{R} 18) / 4 .
\end{array}\right.
$$

\subsection{Smooth hue transition interpolation algorithm for original data}

One of the key objections of the bilinear interpolation is that the hues of adjacent pixels change abruptly and in an unnatural manner. The Bayer CFA pattern, on the other hand, can be thought of as consisting of a luminance channel (the relatively numerous green pixels) and a chrominance channel (the relative sparse red and blue pixels). A scheme can be created to interpolate these channels differently. According to the Mondrian model, the colour ratio rule of adjacent pixels $(m, n)$ and $(i, j)$ can be defined as:

$$
\left\{\begin{array}{l}
\mathrm{R}_{m n} / \mathrm{G}_{m n}=\mathrm{R}_{i j} / \mathrm{G}_{i j}, \\
\mathrm{~B}_{m n} / \mathrm{G}_{m n}=\mathrm{B}_{i j} / \mathrm{G}_{i j} .
\end{array}\right.
$$

- Interpolation of green pixels

In this algorithm, the method for obtaining green pixel values is the same as bilinear interpolation. Interpolated green pixel values is in front of the interpolate pixel values red and blue.

\section{- Interpolation of blue pixels}

The idea here is to try to impose a smooth transition in hue from pixel to pixel. To do so, we define the blue "hue value" and red "hue value" in (6).

$$
\left\{\begin{array}{c}
\text { blue hue }=\mathrm{B} / \mathrm{G}, \\
\text { red hue }=\mathrm{R} / \mathrm{G} .
\end{array}\right.
$$

Considering the interpolation of blue pixel values: there are three different cases of blue pixel value interpolations.

We can estimate blue pixel values at the green position and adjacent blue pixels on left and right. For example, in Fig. 4(a), we can obtain the values of B13 and at G13: 
We can estimate blue pixel value at the green position and adjacent blue pixels on top and bottom. For example, in Fig. 4(a), we can obtain the values of B7 and at G7:

(8)

$$
\mathrm{B} 7=(0.5 \mathrm{G} 7) \times(\mathrm{B} 2 / \mathrm{G} 2+\mathrm{B} 12 / \mathrm{G} 12) \text {. }
$$

We can estimate blue pixel values at the red position. For example, in Fig. 4(a), we can obtain the values of B18 at R18:

(9) $\mathrm{B} 18=(0.25 \mathrm{G} 18) \times(\mathrm{B} 12 / \mathrm{G} 12+\mathrm{B} 14 / \mathrm{G} 14+\mathrm{B} 22 / \mathrm{G} 22+\mathrm{B} 24 / \mathrm{G} 24)$.

\section{- Interpolation of red pixels}

There are three different cases of red pixel value interpolations.

Estimating red pixel values in a green position and adjacent to the location of red pixels on the left and right. For example, in Fig. 4(a), we can obtain the values of R7 at G7:

$$
\mathrm{R} 7=(0.5 \mathrm{G} 7) \times(\mathrm{R} 6 / \mathrm{G} 6+\mathrm{R} 8 / \mathrm{G} 8) .
$$

Estimating red pixel values in a green position and adjacent to the location of red pixels in the top and bottom. For example, in Fig. 4(a), we can obtain the values of R13 at G13:

$$
\mathrm{R} 13=(0.5 \mathrm{G} 13) \times(\mathrm{R} 8 / \mathrm{G} 8+\mathrm{R} 18 / \mathrm{G} 18) .
$$

Estimating red pixel values in a blue position. For example, in Fig. 4(a), we can obtain the values of R12 at B12:

$$
\mathrm{R} 12=(0.25 \mathrm{G} 12) \times(\mathrm{R} 6 / \mathrm{G} 6+\mathrm{B} 8 / \mathrm{G} 8+\mathrm{B} 16 / \mathrm{G} 16+\mathrm{B} 18 / \mathrm{G} 18) .
$$

\section{Experimental results and discussion}

In the following experiments, we use a natural colour key and a red little metal bear as original data, and then we apply the bilinear and smooth hue transition interpolation algorithm to restore the colour information of two original images.

\subsection{Bilinear interpolation with four starting pixels models}

We use bilinear interpolation algorithm with four starting pixels models to restore the colour of original key data respectively, which can be seen in Fig. 5 (b), (c), (d), (e) respectively. The results show that the GB model is right for interpolation algorithm, and the restoration results of starting model with BG, GR, RG pixels are not correct, because they cannot restore correct colour of the key.

Besides, we use bilinear interpolation algorithm with four starting pixels model to restore the colour of metal bear as well, which can be seen in Fig. 6 (b), (c), (d), and (e) respectively. The results show that the GB model is right for interpolation algorithm, and the other starting models with BG, GR, RG pixels are not correct, because they can not restore red colour of the metal bear. Therefore, it should be mentioned that the starting pixels model of Bayer CFA is very important for colour restoration. 


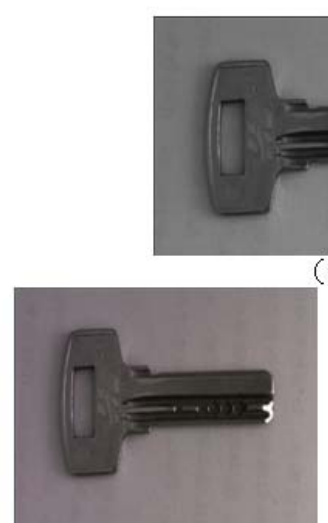

(c)

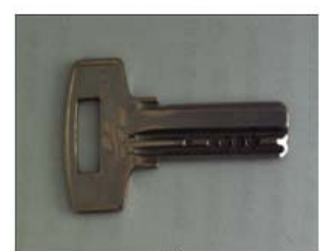

(b)

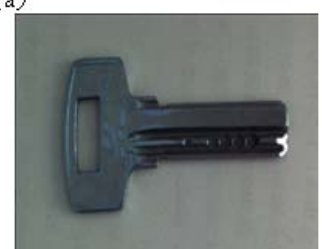

(d)

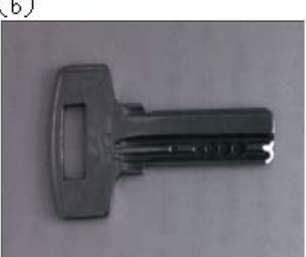

(e)

Fig. 5. The original data of a key (a); bilinear interpolation in accordance with GB starting model (b); BG starting model (c); GR starting mode (d); RG starting model (e)

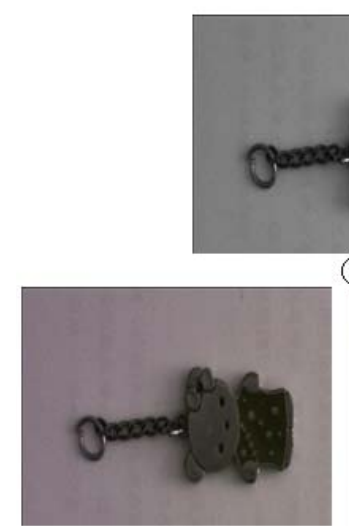

(c)

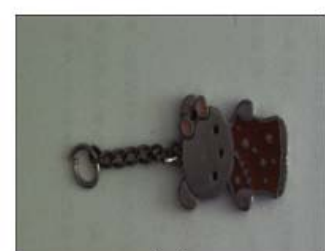

(b)

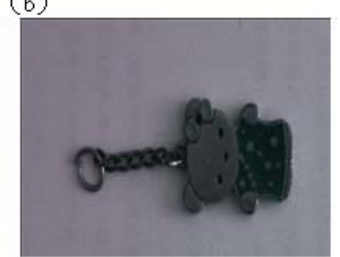

(e)

Fig. 6. The original data of a little metal bear (a); bilinear interpolation in accordance with GB starting model (b); BG starting model (c); GR starting mode (d); RG starting model (e)

4.2. Smooth hue transition interpolation with four starting pixel models

We use smooth hue transition interpolation algorithm with GB, BG, GR, RG starting pixel models to restore original key image respectively, which can be seen in Fig. 7 (a), (b), (c), and (d) respectively. The results show that the GB model is right for interpolation algorithm, the others are not correct, because they can not restore the natural colour of the key.

Besides, according to the starting model with GB, BG, GR, RG pixels, we use smooth hue transition interpolation algorithm to restore the colour of metal bear, which can be seen in Fig. 8 (a), (b), (c), and (d) respectively. The results show that the GB model is also right for interpolation algorithm, and the restoration results of starting model with BG, GR, RG pixels are not correct. 

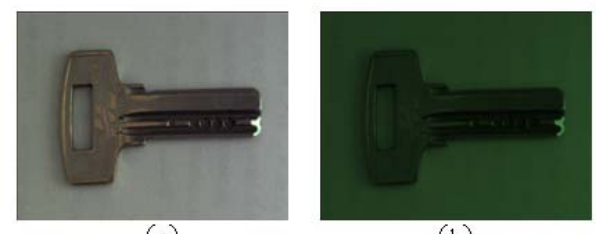

(a)
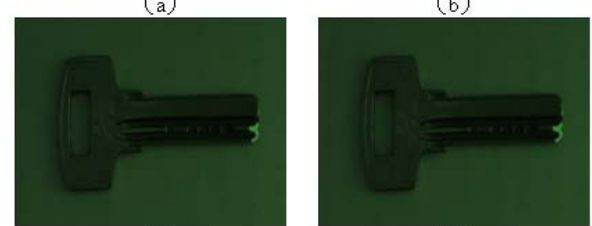

(c)

(d)

Fig. 7. Smooth hue transition interpolation in accord with: GB starting model (a); BG starting model (b); GR starting mode (c); RG starting mode (d)
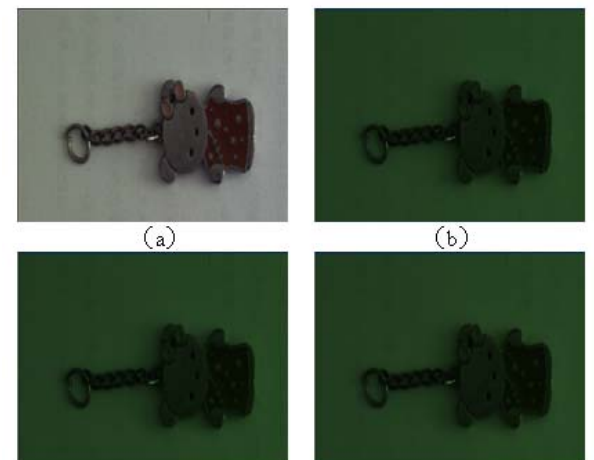

(c)

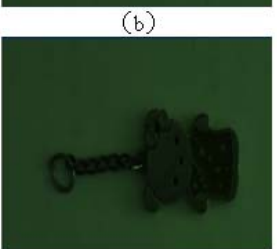

(d)

Fig. 8. Smooth hue transition interpolation in accordance with: GB starting model (a); BG starting model (b); GR starting mode (c); RG starting mode (d);

4.3. Compare the local magnified image of bilinear and smooth hue transition interpolation algorithm

In Fig. 9, we draw three red box areas named “1”, “2”, and “3”, then we zoom in these areas of bilinear and smooth hue transition interpolated image respectively, which can be seen in Fig. 10 and Fig. 11, the results show that the visual effect of restored image by using smooth hue interpolation algorithm is better than that of using bilinear interpolation algorithm.

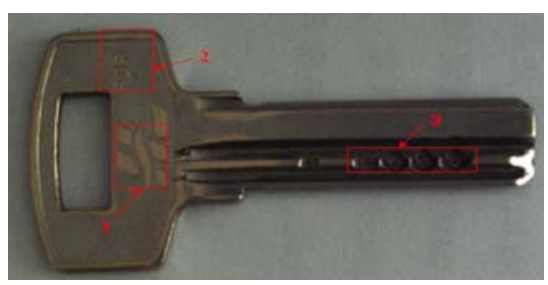

(a)

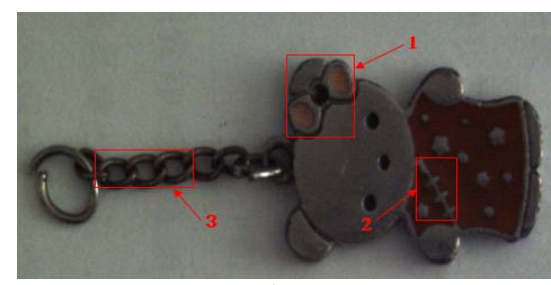

(b)

Fig. 9. Three areas drawn by red box of original: key image (a); little metal bear image (b) 


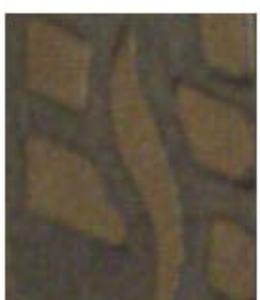

(a)

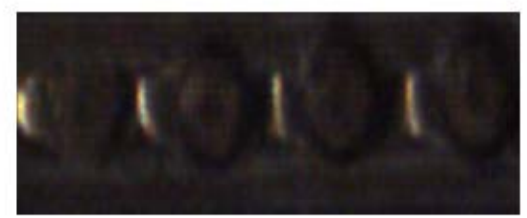

(e)

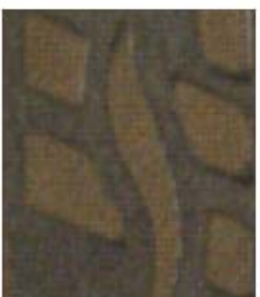

(b)

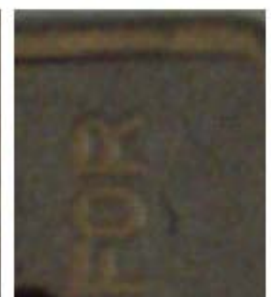

(c)

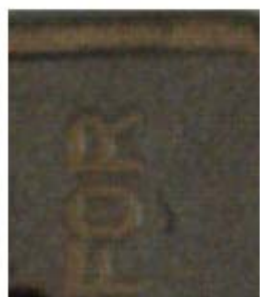

(d)

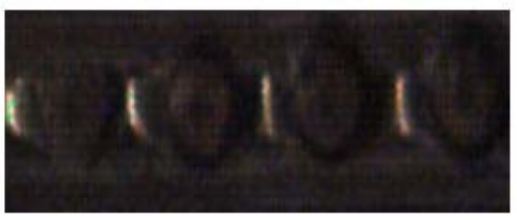

(f)

Fig. 10. Zoom in the area "1" of restored colour key image using: bilinear transition interpolation (a), and smooth hue transition interpolation (b); the area "2" using: bilinear transition interpolation (c), and smooth hue transition interpolation (d); the area " 3 " using: bilinear transition interpolation (e), and smooth hue transition interpolation (f)

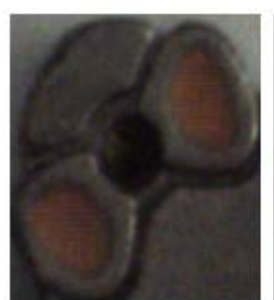

(a)

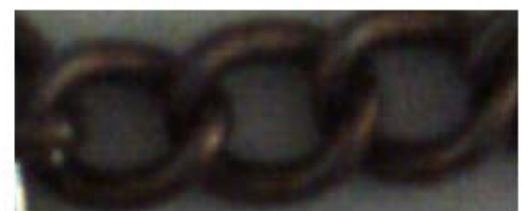

(e)

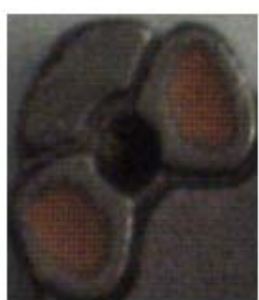

(b)

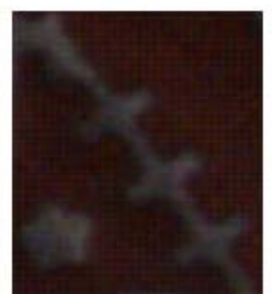

(c)

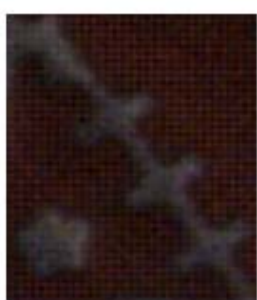

(d)

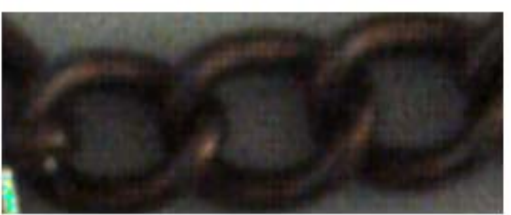

(f)

Fig. 11. Zoom in the area named "1" of restored color metal bear image by using: bilinear interpolation (a), and smooth hue transition interpolation (b); zoom in the area named "2" by using: bilinear interpolation (c), and smooth hue transition interpolation (d); zoom in the area named " 3 ” by using: bilinear interpolation (e), and smooth hue transition interpolation (f)

\subsection{Objective quality measure}

To compare these algorithms objectively, we adopt Mean Square Error (MSE) as our quality metrics. The MSE between the original image $I_{0}(x, y)$ and the interpolated image $I_{\mathrm{i}}(x, y)$ for each colour channel is defined in (13), where $m \times n$ is the size of the image. 


$$
\mathrm{MSE}=\frac{\sum_{x=1}^{m} \sum_{y=1}^{n}\left[I_{0}(x, y)-I_{\mathrm{i}}(x, y)\right]^{2}}{m \times n} .
$$

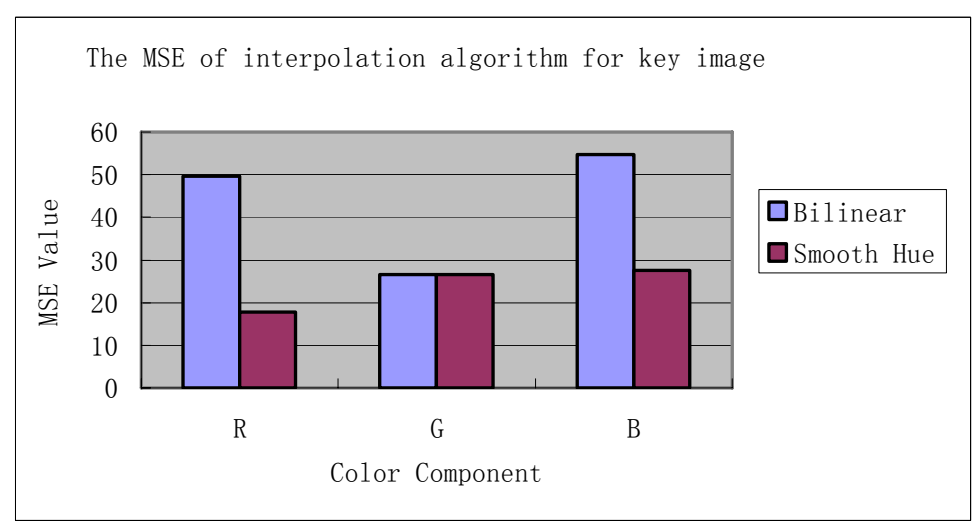

(a)

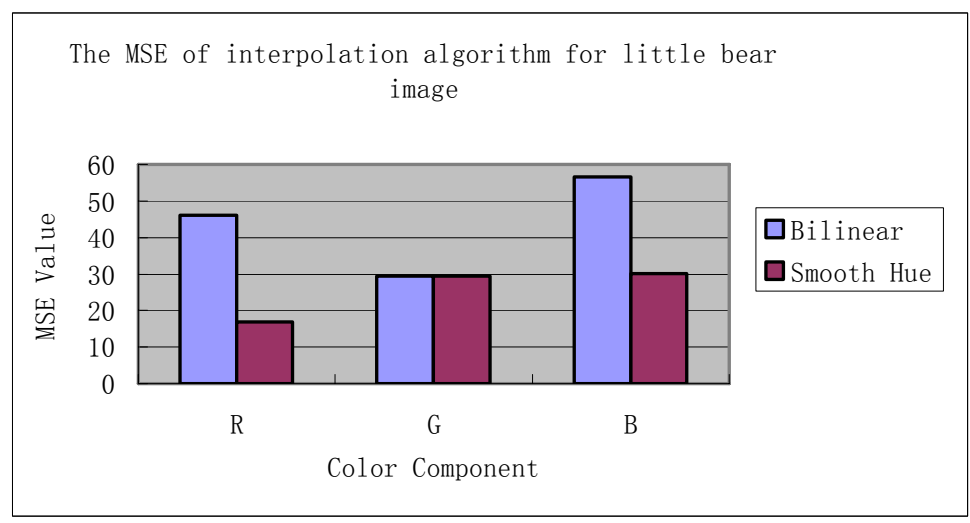

(b)

Fig.12. The mean square error between the original image and the interpolated "key" image at R, G and $\mathrm{B}$ channel (a); the mean square error between the original image and the interpolated little metal bear image at R, G and B channel (b)

We can see that the MSE of restored colour information by using smooth hue transition interpolation algorithm is lower than that of the bilinear interpolation algorithm in Fig. 12, thus, the restored image by smooth hue transition interpolation is closer to the original image.

\section{Conclusion}

In this paper, we apply a Bayer CFA with four starting pixels, and then we use bilinear interpolation algorithm and smooth hue transition interpolation algorithm to restore the colour information of original image, and the smooth hue transition interpolation can obtain more colour details and enhance the edge of original image. 


\section{References}

1. Gunturk, B. K., J. G l o t z b a c h. Demosaicking: Color Filter Array Interpolation. - IEEE Signal Processing Magazine, Vol. 22, 2005, No 1, 44-54.

2. S a k a m o t o, T. Software Pixel Interpolation for Digital Still Cameras Suitable for a 32-bit MCU. - IEEE Transactions on Consumer Electronics, Vol. 44, 1998, No 4, 1342-1352.

3. X i a o s o n g, L., X. Y a n g, J. W e n, J. W a n g. A Color Interpolation Method for Digital Image Sensors. - Journal of Image and Graphics, Vol. 8(A), 2003, No 5, 516-521.

4. L i, Q., H. F e n g, Z. X u. A Color Interpolation Algorithm of CCD Based on Green Components. - Journal of Optoelectronics Laser, Vol. 12, 2001, No 8, 842-844.

5. Z hou, R., J. H e, Z. H on g. Adaptive Algorithm of Auto White Balance for Digital Camera. Journal of Computer-Aided Design \& Computer Graphics, Vol. 17, 2005, No 3, 529-533.

6. Parulski, K. A. Color Filters and Processing Alternatives for One-Chip Cameras. - IEEE Transactions on Electron Devices, Vol. 32, 1985, No 8, 1381-1389.

7. H i d e m o r i, Z. A New Digital Signal Processor for Progressive Scan CCD. - IEEE Transactions on Consumer Electronics, Vol. 44, 1998, No 2, 289-295.

8. Kom at su, T., K. A i z a wa, T. I g a r a s i. Signal-Processing Based Method for Acquiring Very High Resolution Image with Multiple Cameras and Its Theoretical Analysis. - Proc. Inst. Elec. Eng, Vol. 140, 1993, No 1, 19-25.

9. Patti, A. J., Y. Alt un b a s a k. Artifact Reduction for Set Theoretic Super Resolution Image Reconstruction with Edge Adaptive Constraints and Higher-Order Interpolants. - IEEE Transactions on Image Processing, Vol. 10, 2001, No 1, 179-186.

10. E l a d, M., A. F e u e r. Restoration of a Single Super-Resolution Image from Several Blurred, Noisy and Undersampled Measured Images. - IEEE Transactions on Image Processing, Vol. 6, 1997, No 12, 1646-1658. 